\title{
El patrimonio arqueológico subacuático en la evaluación de impacto ambiental
}

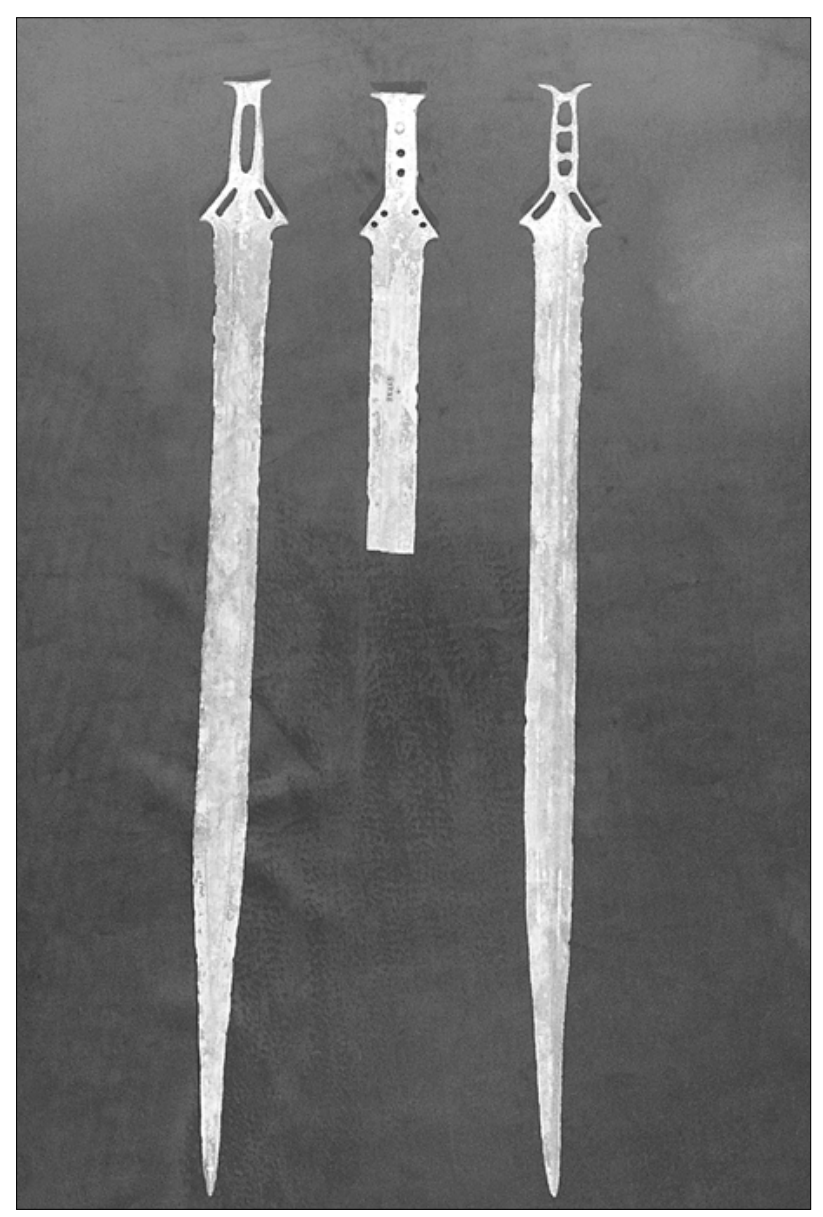

\section{Milagros Alzaga García}

Centro de Arqueología Subacuática del IAPH

\section{Resumen}

Aunque son numerosos los agentes que inciden negativamente sobre el Patrimonio Arqueológico Subacuático, son aquellos de carácter antrópico los que mayor repercusión tienen sobre nuestro patrimonio sumergido.

A pesar de contar con una normativa legal clara y precisa para la protección del Patrimonio arqueológico en general y del subacuático en particular, no se puede negar que existe una reticencia, en la mayoría de los casos, a la hora de aplicar las cautelas oportunas para su correcta investigación y conservación.

\section{Palabras claves}

Evaluación de impacto ambiental, Patrimonio Arqueológico Subacuático, Gestión, Legislación, Protección.

\section{Introducción}

El impacto de las actividades humanas sobre el medio ambiente no es un fenómeno exclusivo de nuestro tiempo, aunque si se puede afirmar que el gran detonante, en este sentido, fue la revolución industrial que dio lugar al inicio de los grandes cambios en la agricultura, la industria y el transporte. Como consecuencia, empezaron ha surgir grupos medioambientales -en el Reino Unido y Estados Unidos-, cuyos objetivos principales eran la protección y conservación del medio ambiente en general. 
En este ámbito, y dentro de los bienes históricos, en el año 1877, en Gran Bretaña, inició su andadura la Sociedad Protectora de Edificios Antiguos como respuesta a los problemas, ya patentes en esos momentos, de expansión urbana.

Los desarrollos tecnológicos del siglo $\mathrm{XX}$ aumentaron la inquietud de los movimientos medioambientales con el objetivo de proteger los espacios naturales, las especies vegetales y animales así como los edificios amenazados (HUNT, D; JOHNSON, C. 1997).

Así comenzaron a surgir las primeras iniciativas gubernamentales, entre las cuales debe destacarse, desde el punto de vista arqueológico, la Ley del 12 de marzo de 1930 que protegía, en Francia, los denominados sites definidos por la UNESCO como varios tipos de lugares interesantes: urbanos, rurales, mixtos (obra a la vez de la naturaleza y del hombre) y también científicos o arqueológicos (GIOLITTO, P. 1984).

Si bien estos movimientos quedaron ralentizados durante los años de postguerra se vieron relanzados, con más fuerza aún si cabe, durante los años 70, lo que dio lugar a un aumento de las acciones gubernamentales desde el punto de vista legislativo.

Sin embargo, casi todas las acciones han omitido, en la mayoría de las ocasiones, las repercusiones de las actividades humanas sobre los yacimientos arqueológicos en general. Esta circunstancia hizo que en el año 1989 el Consejo de Europa, en su reunión de Estrasburgo del 13 de abril, redactara "unas recomendaciones a los estados miembros relativas a la protección y puesta en valor del Patrimonio Arqueológico en el contexto de las operaciones urbanísticas de ámbito urbano y rural" (Muñoz Vicente, A. 1997). Ante estos datos, se puede comprobar que los yacimientos arqueológicos localizados en el medio subacuático quedaron fuera del marco de protección antes citado, circunstancia ésta que se contrapone al informe Brundtland emitido por la Comisión Mundial sobre Medio Ambiente y Desarrollo, al definir el concepto de DesarroIlo Sostenible como aquel que satisface las necesidades del presente sin comprometer la capacidad de las generaciones futuras para satisfacer las suyas propias (HUNT, D. y JOHNSON, C. 1997). (Foto I)

Esta carencia quizás puede estar motivada por la falta de una tradición investigadora dentro del ámbito de la arqueología subacuática lo que ha incidido de manera negativa sobre la ausencia de gestión en esta materia.

En este sentido, la Comunidad Autónoma de Andalucía, por medio de Instituto Andaluz del Patrimonio Histórico, cuenta con el Centro de Arqueología Subacuática, que contempla dentro de sus funciones la documentación, protección, investigación, intervención, conservación y difusión del Patrimonio Arqueológico Sumergido (PAS).

Dentro del ámbito de la protección del PAS hay que incidir en el hecho de que los yacimientos arqueológicos en general y los subacuáticos en particular pose- en unas características y necesidades específicas, por lo que existe la prioridad de formar a técnicos en el ámbito de la gestión de este patrimonio.

\section{Aspectos legislativos}

Desde la promulgación de la Constitución en el año 1978, en España se inicia un proceso de transferencias de competencias a las Comunidades Autónomas siendo, en consecuencia, estas últimas las competentes en materia de medio ambiente, por medio de sus correspondientes Estatutos de Autonomía.

En el caso de la Comunidad Autónoma de Andalucía son la Ley 7/1994, de 18 de mayo, de Protección Ambiental y el Decreto 292/1995 que la reglamenta las que vienen a complementar las siguientes normativas:

- la Directiva 85/337 del Consejo de las Comunidades Europeas de 27 de junio de 1985, que establece la obligatoriedad de someter determinados proyectos de obras, instalaciones o actividades a un procedimiento reglado de Evaluación de Impacto Ambiental, con carácter previo a su aprobación y ejecución.

- el Real Decreto Legislativo I302/1986, de 28 de junio, de Evaluación de Impacto Ambiental, que constituye la norma básica en esta materia en todo el territorio nacional.

- el Real Decreto ||31//988, de 30 de septiembre, que desarrolla el anterior. (HUNT, D. y JOHNSON, C. 1997).

Esta Ley (7/1994) articula, por medio de título II artículo 8, la prevención ambiental a través de las siguientes medidas:

I.- Evaluación de Impacto Ambiental.

2.- Informe Ambiental.

3.- Calificación Ambiental.

En el campo de la arqueología en general y de la arqueología subacuática en particular, es la evaluación de impacto ambiental la que puede resultar de gran utilidad para poder llevar a buen término la acción preventiva sobre los yacimientos, ya que tal y como se indica en el artículo 20.1 las actuaciones sujetas a Evaluación de Impacto Ambiental comprendidas en el artículo 5. I. de esta Ley no deberán autorizarse o ejecutarse sin haberse complementado dicho procedimiento, o en contra de lo previsto en la Declaración de Impacto Ambiental.

De esta forma, estarán sometidas a este proceso las siguientes obras que inciden en el medio subacuático, y que se encuentran reflejadas en el Anexo I de la Ley:

- refinerías de petróleo bruto.

- almacenamiento permanente o eliminación de residuos radioactivos.

- plantas siderúrgicas integrales. 


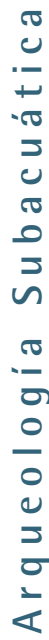

Fotos 2 y 3

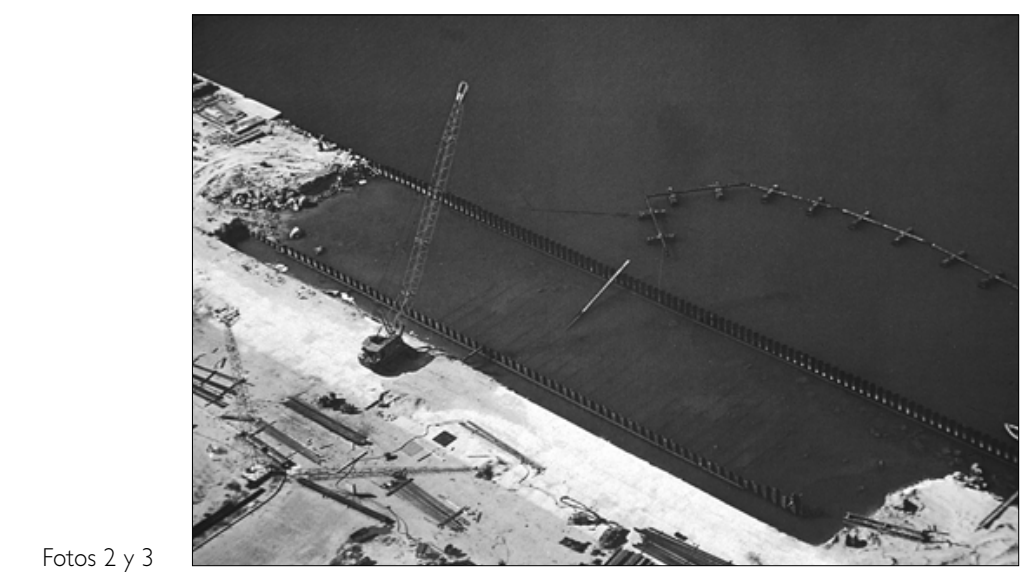
292/1995 se definen las obras marítimo-terrestres como las estructuras marítimas necesarias para las obras de defensa, mejora y recuperación de la costa, tales como diques y espigones, sean éstos perpendiculares o paralelos a la costa, emergidos, semisumergidos o sumergidos, siempre que superen los 12 metros de longitud.

- oleoductos y gaseoductos, definidos en el Decreto 292/1995 como las de nueva planta, incluyendo las instalaciones necesarias para el tratamiento, manipulación o almacenamiento de productos intermedios.

- actividades de relleno, drenaje y desecación de zonas húmedas. (Foto 3)
Asimismo en el Decreto 292/1995, anteriormente citado, se hace una relación más detallada, dentro del punto 15 de su anexo, de algunas de las obras marítimo-terrestres que quedan sujetas a este Reglamento, como son:

- La construcción de emisarios submarinos para el vertido de aguas residuales urbanas o industriales al mar.

- Las obras de explotación de los yacimientos submarinos de arena siempre que el volumen total aprovechable supere los tres millones de metros cúbicos.

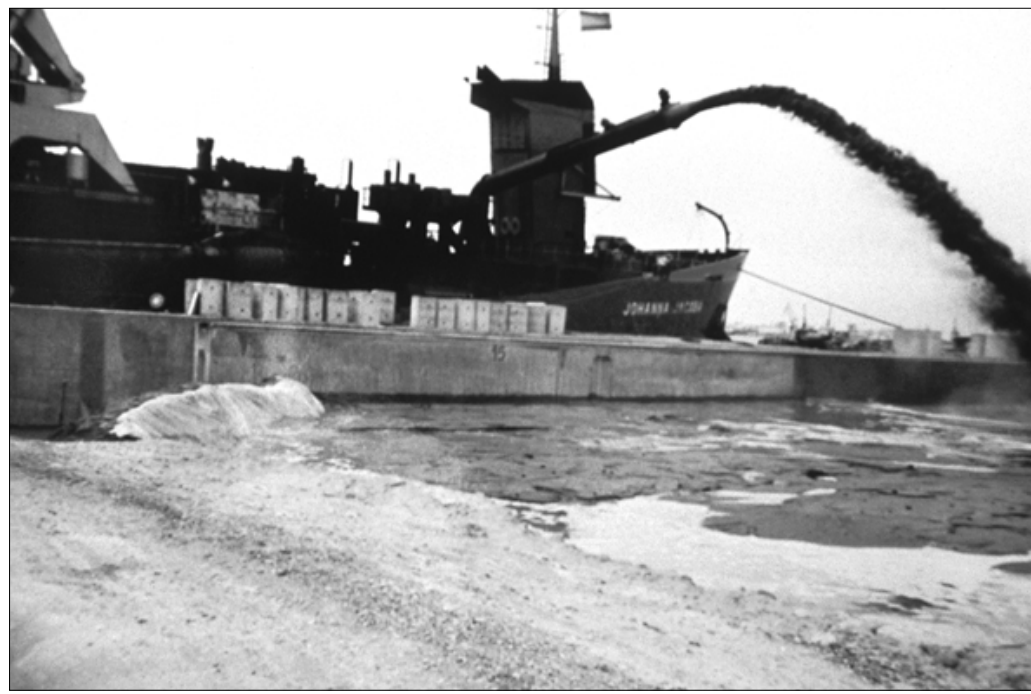

- Las aportaciones de arenas a la costa para la mejora, recuperación, regeneración o creación de playas, cuando superen la cantidad de un millón de metros cúbicos.

- Las obras de muros, revestimientos y escolleras en el borde del mar, siempre que estén situadas en tramos de costa constituidos por materiales sueltos, y que estén en contacto con el agua del mar. (Foto 4)

Corresponde a la Consejería de Medio Ambiente el recabar toda la información necesaria para poder emitir la Declaración de Impacto Ambiental de los distintos proyectos anteriormente enunciados. En consecuencia, tal y como se contempla en la Ley |/9| de 3 de julio, de Patrimonio Histórico de Andalucía, la Dirección General de Bienes Culturales de la Consejería de Cultura deberá recibir toda aquella información que se requiera para poder así dictaminar las cautelas arqueológicas necesarias para la protección del Patrimonio Histórico Andaluz.

Para poder dictaminar las medidas de protección adecuadas la Consejería de Cultura puede tomar como base la siguiente legislación:

I. La Ley $1 / 91$ de 3 de julio, de Patrimonio Histórico de Andalucía donde, en sus artículos 47 y 48 , queda recogida una nueva figura de protección del Patrimonio Arqueológico: la "Zona de Servidumbre Arqueológica". Se señala que "el planeamiento territorial o urbanístico, así como los planes y programas de actuación que afecten a Zonas de Servidumbre Arqueológica precisarán el informe favorable de la Consejería de Cultura y Medio Ambiente, quien deberá emitirlo una vez que los documentos hayan adoptado su redacción final y antes de ser sometidos a aprobación definitiva" (Art. 49.2). Este mismo artículo en su punto 3 indica que la realización de obras de edificación o cualquiera otra actuaciones que lleven aparejadas la remoción de terrenos en Zonas de Servidumbre Arqueológica se notificará a la Consejería de Cultura y Medio Ambiente con un mínimo de quince días de anticipación. Durante este plazo dicha Consejería podrá ordenar la realización de catas o prospecciones arqueológicas.

Con respecto a las Evaluaciones de Impacto Ambiental el artículo I 4.3 señala que "en la tramitación de evaluaciones de impacto ambiental de actuaciones que puedan afectar directa o indirectamente a bienes inte- 
grantes del Patrimonio Histórico Andaluz la Agencia del Medio Ambiente recabará informe de la Dirección General de Bienes Culturales e incluirá en la declaración de impacto ambiental las consideraciones o condiciones resultantes de dicho informe". Este artículo se ve reforzado por el 60.2 donde se establece que "para la evaluación de impacto ambiental de actuaciones que afecten a Zonas de Servidumbre Arqueológica o Zonas Arqueológicas se recabará informe de la Consejería de Cultura y Medio Ambiente, incluyéndose sus observaciones o condiciones en la declaración de impacto ambiental", el cual se ve a su vez complementado por el artículo 77 del Decreto 19/1995, de 7 de febrero, por el que se aprueba el Reglamento de Protección y Fomento del Patrimonio Histórico de Andalucía, por el cual se debe "incluir un inventario de los bienes de carácter arqueológico que puedan verse afectados por la ejecución del proyecto" dentro de los estudios de impacto ambiental.

2. La Ley $22 / 1988$, de 28 de julio de Costas sobre la base de sus artículos:

- Artículo 2: afirma que la actuación administrativa sobre el dominio público marítimo-terrestre perseguirá los siguientes fines: regular la utilización racional de estos bienes en términos acordes con su naturaleza, sus fines y con el respeto al paisaje, al medio ambiente y al PATRIMONIO HISTÓRICO.

- Artículo 42.2.: cuando las actividades proyectadas pudieran producir una alteración importante del dominio público marítimo-terrestre se requerirá además una previa evaluación de sus efectos sobre el mismo, en la forma que se determine reglamentariamente.

- Artículo 63: para otorgar las autorizaciones de extracciones de áridos y dragados, será necesaria la evaluación de sus efectos sobre el dominio público marítimo-terrestre, referida tanto al lugar de extracción o dragado como al de descarga en su caso. (Foto 5)

3. El Real Decreto 147//1989, de I de diciembre, por el que se aprueba el Reglamento General para el desarrollo y ejecución de la Ley antes enunciada, basándose en los artículos:

- Artículo 206.2: se indica que "las funciones de la Administración del Estado en el mar territorial, aguas interiores, zona económica y plataforma continental en materia de defensa, pesca y cultivos marinos, salvamento, lucha contra la contaminación, seguridad de la vida humana en el mar, extracciones de restos, PROTECCIÓN DEL PATRIMONIO ARQUEOLÓGICO ESPAÑOL, investigación y explotación de recursos y otras no reguladas en la Ley de Costas y este Reglamento, se ejercerán en la forma y por los Departamentos u Organismos que las tuvieran encomendadas a la entrada en vigor de la Ley de Costas, sin perjuicio de lo establecido en su legislación específica o en los convenios internacionales que, en su caso, sean de aplicación. (Fotos 6 y 7 )
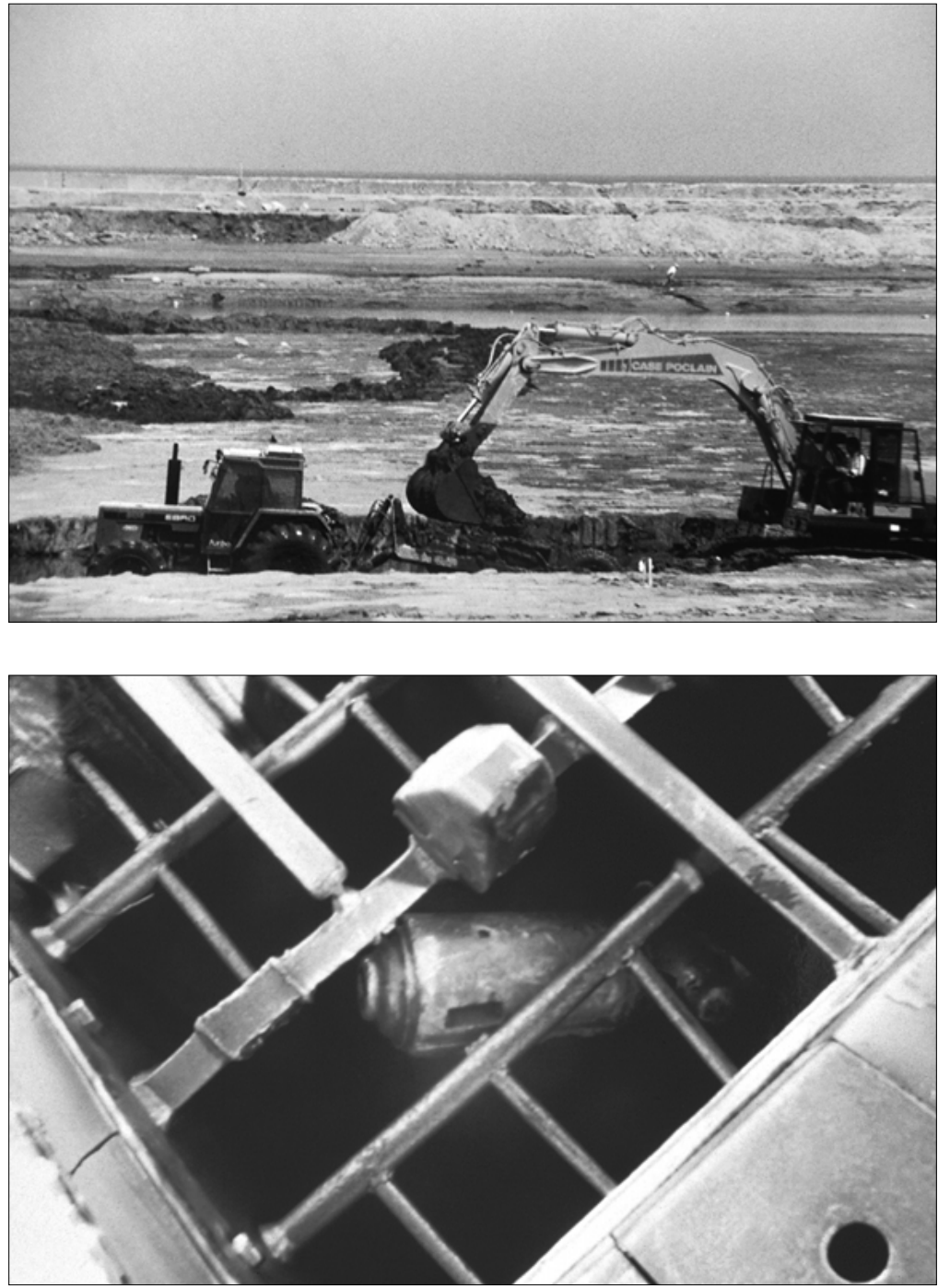

Fotos 4 y 5

- Artículo 207.l: se indica que serán las Comunidades Autónomas las que ejerzan las competencias que en las materias de ordenación territorial y del litoral, puertos, urbanismo, vertidos al mar y demás relacionadas con el ámbito de la Ley de Costas tengan atribuidas en virtud de sus respectivos Estatutos.

4. Real Decreto III2/1992, de 18 de septiembre, por el que se modifica parcialmente el Reglamento General para desarrollo y ejecución de la Ley 22/1988, con el objeto de adecuarlo al régimen de competencias que corresponden a la administración del Estado y a las Comunidades Autónomas en el espacio litoral, de conformidad con las citadas sentencias.

5. Ley 27/1992, de 24 de noviembre, de Puertos del Estado y de la Marina Mercante.

- Artículo 2: los proyectos de ampliación que modifiquen sustancialmente la configuración y los límites exteriores de los puertos estatales deberán incluir un estudio de impacto ambiental y se sujetarán al procedimiento de declaración de impacto ambiental 

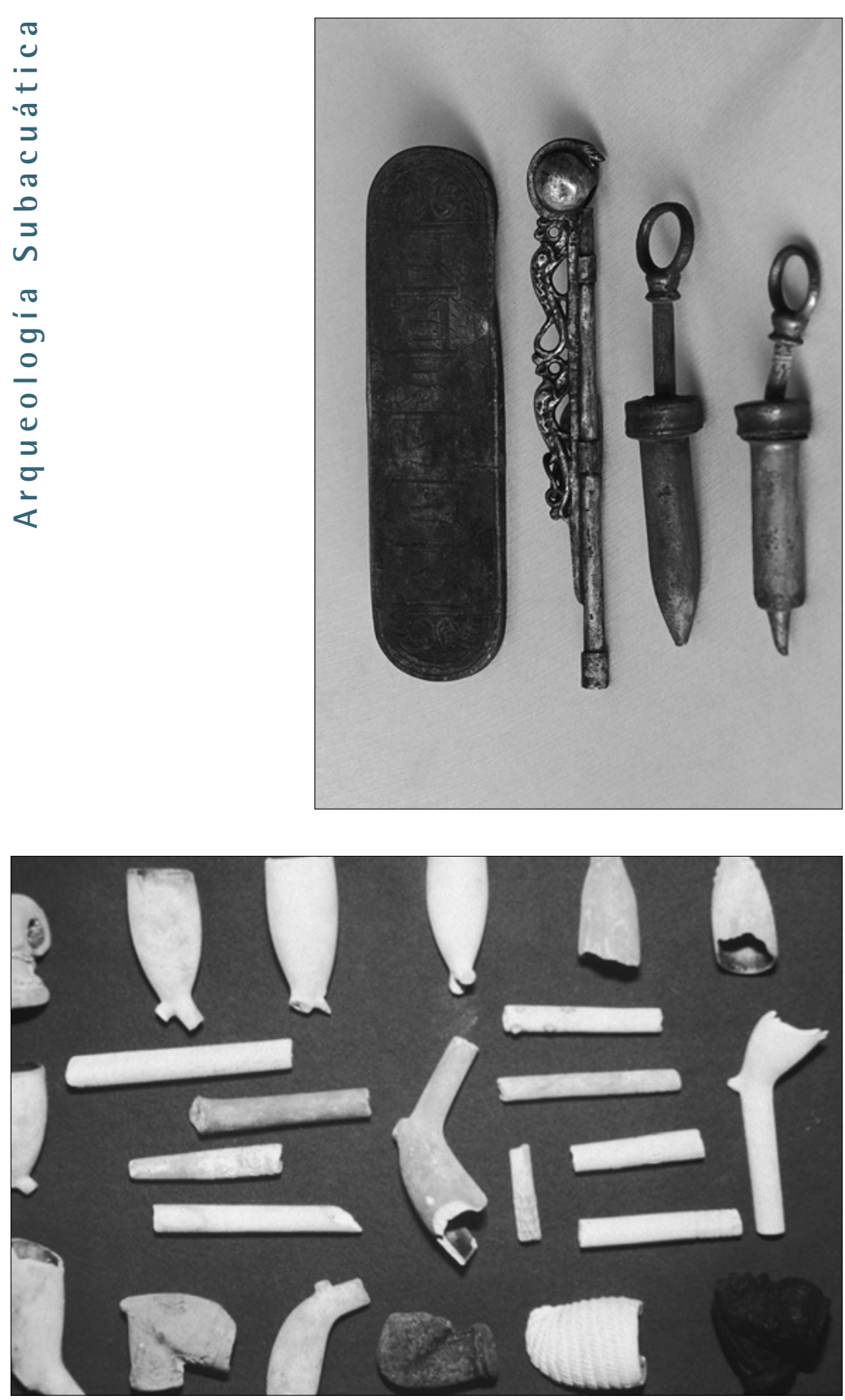

Fotos 6 y 7

cuando por la importancia de la actuación sean susceptibles de modificar o alterar, de forma notable, el medio ambiente.

- Artículo 62: los proyectos de dragados portuarios, incluso los ejecutados por la Autoridad Portuaria, incluirán un estudio de evaluación de sus efectos sobre la dinámica litoral y la biosfera marina, así como, cuando proceda, sobre la posible localización de restos arqueológicos. Se solicitará informe de las Administraciones competentes en materia de pesca y de arqueología.

6. Ley $62 / 1997$, de 26 de diciembre, de modificación de la Ley 27/1992, de 24 de noviembre, de Puertos del Estado y de la Marina Mercante. Esta Ley, similar en sus artículos a la citada en el punto 6, establece las medidas precisas para facilitar que las Comunidades Autónomas participen con mayor intensidad en la estructura organizativa de las Autoridades Portuarias.

\section{Contenidos arqueológicos que debe contemplar la evaluación de impacto ambiental}

Siguiendo el Reglamento de Evaluación de Impacto Ambiental de la Comunidad Autónoma Andaluza (Decreto 292/1995, de 12 de diciembre), se pueden transpolar los contenidos del estudio de impacto ambiental, descritos en el artículo II, a los contenidos de estudio de impacto arqueológico, tomando como base las obras que afectan al medio subacuático, cuyo resultado bien podría ser el siguiente:

\section{Descripción del proyecto y sus acciones}

A) LOCALIZACIÓN: situación exacta del lugar donde va a tener lugar la obra.

B) ACCIONES SUSCEPTIBLES DE PRODUCIR UN IMPACTO: analizar la obra con el objeto de comprobar qué repercusiones puede ocasionar sobre el patrimonio arqueológico sumergido.

C) DESCRIPCIÓN DE LOS MATERIALES A UTILIZAR, SUPERFICIE A OCUPAR: tipos de dragas (cortadora, de succión, de succión en marcha autoportante, de cuchara, de cangilones), gánguiles, zonas de vertido, forma de propulsión de la arena en el caso de tratarse de una regeneración de playa o del relleno de una zona, así como los materiales a utilizar para la construcción de espigones, ampliación de muelles, emisarios, etc.

D) ESTIMACIÓN DE LOS AGENTES QUE VAN A INCIDIR NEGATIVAMENTE SOBRE LOS YACIMIENTOS

\section{E) ALTERNATIVAS: razonarlas}

F) DOCUMENTACIÓN CARTOGRÁFICA: las escalas que se indican son las mínimas

- Plano de situación 1:50.000

- Plano de emplazamiento I:10.000

- Plano general y de detalle de la obra

\section{Inventario Arqueológico}

A) ESTUDIO DEL ESTADO DEL LUGAR DESDE EL PUNTO DE VISTA ARQUEOLÓGICO: antecedentes de las obras y actuaciones arqueológicas llevadas a cabo en la zona, documentación existente sobre el área en la que se va a actuar o sobre los posibles yacimientos arqueológicos que en ella pudieran existir.

B) DESCRIPCIÓN DE LA CLASIFICACIÓN DE LOS YACIMIENTOS DESDE EL PUNTO DE VISTA LEGAL: situación legal de la zona, explicar el tipo de figura que la protege, base de datos en la que se pueden localizar los yacimientos arqueológicos: 
- ARQUEOS base de datos que recoge el Inventario de Yacimientos Arqueológicos de Andalucía.

- YACSUB compuesta principalmente por noticias orales de buceadores, pescadores y gentes del mar, donde se hacía referencia a la existencia de restos arqueológicos.

C) RELACIÓN DE LA NORMATIVA LEGAL: legislación en la que puede basarse la evaluación de impacto ambiental (Ley |/9| de 3 de julio, de Patrimonio Histórico de Andalucía; Decreto 18/1995, de 7 de febrero, por el que se aprueba el Reglamento de Protección y Fomento del Patrimonio Histórico de Andalucía; Ley 7/1994 de 18 de mayo, de Protección Ambiental; Decreto 292//995 de 7 de febrero por el que se aprueba el Reglamento de Evaluación de Impacto Ambiental de la Comunidad Autónoma de Andalucía; Ley 22/1988 de 28 de julio, de Costas; Real Decreto |47|//989 de I de diciembre, que reglamenta la ley anteriormente citada; Real Decreto | | |2/1992 de I 8 de septiembre, por el que se modifica parcialmente el Reglamento de 1989; Ley 27/1992 de 24 de noviembre, de Puertos del Estado y de la Marina Mercante, así como la Ley 62/1997 de 26 de diciembre, que modifica la del 92).

\section{Identificación y valoración de los impactos}

A) IDENTIFICACIÓN DE LOS EFECTOS: analizar el grado de incidencia que los distintos agentes pueden causar sobre la zona y los yacimientos.

B) SEPARAR LOS EFECTOS POSITIVOS DE LOS NEGATIVOS, LOS TEMPORALES DE LOS PERMANENTES Y LOS REVERSIBLES DE LOS IRREVERSIBLES

C)SEPARAR LOS EFECTOS A CORTO, MEDIO Y LARGO PLAZO

\section{Propuestas de medidas protectoras y conectoras}

A) MEDIDAS PREVISTAS PARA SUPRIMIR O ATENUAR LOS EFECTOS: tipo de cautelas arqueológicas que se proponen.

B) ACCIONES A COMPENSAR DICHOS EFECTOS

Planos generales y detalle de las medidas propuestas.

\section{Programa de vigilancia}

A) DEFINICIÓN DE LOS TIPOS Y OBJETIVOS DEL CONTROL ARQUEOLÓGICO: Se reflejarán las actuaciones arqueológicas subacuáticas que se proponen, pudiendo aconsejarse distintas alternativas técnicas y metodológicas, así como los objetivos que persiguen.

B) ANÁLISIS Y VIABILIDAD DEL PROGRAMA PROPUESTO (Plazos, período, personal)

C) PROPUESTA PARA LA ELABORACIÓN DE INFORMES (Tipo y período de emisión)

\section{Documento de síntesis}

\section{Conclusiones}

Como ha quedado reflejado en páginas precedentes, las evaluaciones de impactos ambientales pueden ser consideradas como la herramienta idónea para la salvaguarda y protección del Patrimonio Arqueológico Subacuático andaluz, al contemplar los informes arqueológicos como forma de controlar los efectos que, las distintas obras realizadas en el medio subacuático, puedan causar sobre los diversos yacimientos.

No obstante, puede apreciarse que en este punto la Consejería de Cultura fundamenta los criterios de protección más por las legislaciones que le son ajenas (Ley de Costas, Ley de Puertos del Estado y de la Marina Mercante), que por aquellas que le son intrínsecas. Esta circunstancia debería ser solventada llevándose a cabo una revisión de la legislación actual con el fin de adaptarla a las nuevas necesidades que demanda la sociedad.

Otro punto importante que debería tenerse presente es el de la colaboración que debe existir entre las distintas administraciones tanto autonómicas como estatales, tal y como queda claramente recogido en el artículo 16 del Decreto 292/1995, de 12 de diciembre por el que se aprueba el Reglamento de Evaluación de Impacto Ambiental de la Comunidad Autónoma de Andalucía, así como en el artículo 209 del Real Decreto $|47| / \mid 989$, de I de diciembre, por el que se aprueba el Reglamento General para el desarrollo y ejecución de la Ley 22/1988, de 28 de julio, de Costas.
AGUILAR FERNÁNDEZ, S. El reto del medio ambiente. Conflictos e intereses en la política medioambiental europea. Madrid. 1997

CANTER, L.W. Manual de Evaluación de Impacto Ambiental. Técnicas para la elaboración de estudios de impacto. Madrid. 1997

GIOLITTO, P. Pedagogía del medio ambiente. Ed. Herder. Barcelona. 1984
HUNTO, D; JOHNSON, C. Sistemas de Gestión Medioambiental. Madrid. 1997

MUÑOZ VICENTE, A. La evaluación del impacto ambiental. Un instrumento efectivo de protección del patrimonio artístico arqueológico en ámbito rural. PH: Boletín del IAPH, n²0, 1997, p.98102. 